\title{
Effects of Localized Muscle Fatigue and Whole Body Fatigue on Postural Control during Single-Leg Stance
}

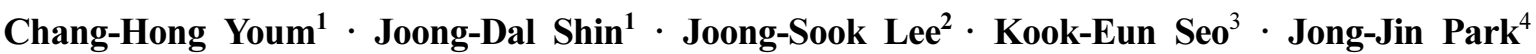 \\ ${ }^{1}$ Department of Coaching, College of Sport Sciences, Dong-A University, Busan, Korea \\ ${ }^{2}$ Division of Physical Education, College of Medical and Life Sciences Silla University, Busan, Korea \\ ${ }^{3}$ Division of Sport Sciences, Pusan National University, Busan, Korea \\ ${ }^{4}$ School of Sports and Health, College of Arts, Kyungsung University, Busan, Korea \\ Received 20 January 2014; Received in revised form 13 March 2014; Accepted 21 May 2014
}

\begin{abstract}
The purpose of this study was to investigate the effects of localized muscle fatigue and whole body fatigue on postural control during single-leg stance after impairment induced by heel raise and the Harvard step test. Thirty-eight university students (19 men: age, $20.1 \pm 0.2 \mathrm{yrs}$; height, $175.0 \pm 5.23 \mathrm{~cm}$; weight, $66.8 \pm 5.6 \mathrm{~kg}$; body mass index, $21.8 \pm 1.7 \mathrm{~kg} / \mathrm{m}^{2}, 19$ women: age, $20.6 \pm 1.1 \mathrm{yrs}$; height, $163.6 \pm 6.7 \mathrm{~cm}$; weight, $58.8 \pm 4.6 \mathrm{~kg}$; body mass index, $22.0 \pm 2.2 \mathrm{~kg} / \mathrm{m}^{2}$ ) were participated in this study. Subjects performed a series of single-leg postural tasks prior to, following, and 24 hours after completing: heel raise or the Harvard step test. This study showed that the root mean squared distance and velocity in the anteroposterior and mediolateral planes of the center of pressure decreased significantly due to heel raise exercise-induced fatigue. Furthermore, the root mean squared distance in the anteroposterior and mediolateral planes, and the $95 \%$ confidence ellipse area of the center of pressure also decreased significantly 24 hours after completing the Harvard step test. In conclusion, this study showed that both heel raise exercise- and Harvard step exercise-induced fatigue affects postural control during single-leg stance in AP and ML planes. Furthermore, this study suggests that changes in the postural control strategy may have occurred after the fatigue protocols during single-leg stance. Also vision can attenuate the postural deficits associated with the fatigues. In order to clarify these results, further studies using other equipment and variables are necessary.
\end{abstract}

Keywords : Muscle Fatigue, Single-leg Stance, Center of Pressure, Heel Raise, Harvard Step Test

\section{Introduction}

Postural control is maintained by the dynamic integration of internal and external forces(Massion, 1994; Yaggie \& McGregor, 2002), which involves harmonious control of the musculoskeletal, visual, vestibular, and somatosensory systems(Hall \& Brody, 1999; Shumway-Cook \& Woollacott, 2000). For the assessment of the postural control, the force platform is primarily utilized, and the center of pressure (COP) is determined to quantify body sway (Winter, 1995). The COP assessments have been used to investigate changes in postural control in a variety of healthy and spe-

This study was supported by the Dong-A University research fund. Corresponding Author: Chang-Hong Youm

Department of Coaching, College of Sports Sciences, Dong-A University, 840 Hadan-dong, Saha-gu, Busan, Korea

Tel : +82-51-200-7830/ Fax : +82-51-200-7805

E-mail: chyoum@dau.ac.kr cial populations (Dolye, Hsiao-Wecksler, Ragan, \& Rosengren, 2007). The single-leg stance is used as a clinical and experimental tool to assess static postural control in various groups (Bohannon \& Leary, 1995; Frzovic, Morris, \& Vowels, 2000), since this stance is required to perform many everyday motor tasks, including turning, climbing stairs, and walking (Jonsson, Seiger, \& Hirschfeld, 2004).

Measurement of postural control is one approach to quantify neuromuscular control (Gribble \& Hertel, 2004b). Fatigue has been shown to have an adverse effect on neuromuscular control (Gribble \& Hertel, 2004a). Moreover, fatigue and postural control deficits can lead to musculoskeletal injury (Gribble \& Hertel, 2004b). This increased postural sway can increase the risk of musculoskeletal injuries such as ankle sprain (Christina, White, \& Gilchrist, 2001; Letafatkar, Alizadeh, \& Kordi, 2009; Rozzi, Lephart, Gear, \& Fu, 1999; Urabe et al., 2005).

Muscle fatigue relates to a decline in performance during high-intensity exercise. The fatigue-induced changes in per- 
formance include reduced force production, decreased shortening velocity, and slowed relaxation (Allen \& Westerblad, 2001). Factors contributing to muscle fatigue are divided largely into 2 groups: the central factor generates fatigue as a result of the disordering of neuromuscular transmission between the central nervous system and the muscular membrane, whereas the peripheral factor leads to alteration within the muscle (Giannesini, Cozzone, \& Bendahan, 2003). Muscle fatigue depends on the type of exercise and its various causes, and its behavior is based on the manner in which it is induced (Giannesini et al., 2003; Letafatkar et al., 2009).

Many researchers have examined the relationship between lower leg muscle fatigue and COP sway (Adlerton \& Moritz, 1996; Cobeil, Blouin, Bégin, Nougier, \& Teasdale, 2003; Gribble \& Hertel, 2004a, 2004b; Shin \& Youm, 2011; Yaggie \& McGregor, 2002; Youm \& Kim, 2012). Lundin, Feuerbach, and Grabiner (1993) studied how postural control during single-leg stance was affected by plantar flexor and dorsiflexor fatigue in 8 healthy men, and found that both mediolateral and anteroposterior sway increased significantly. Similarly, Yaggie and McGregor (2002) investigated how postural control during single-leg stance was impacted by plantar flexor and dorsiflexor fatigue in 24 healthy men, and reported that mediolateral sway increased significantly. Gimmon, Riemer, Oddsson, \& Melzer (2011) reported that localized plantar flexor fatigue caused impairment to postural control in the sagittal plane. Lin et al. (2009) investigated that acute effects of fatigue location and age on changes in postural control induced by localized muscle fatigue, and resulted that the acute effects of fatigue differed between joints. And ankle joint fatigue resulted in significant effects on postural control.

On the other hand, Adlerton and Moritz (1996) examined the effect of calf muscle fatigue on postural control during single-leg stance and found no significant reduction. Gribble and Hertel (2004a) studied the effects of ankle, knee, and hip joint fatigue on postural control during single-leg stance in 4 men and 10 women, and reported that localized muscle fatigue in the anteroposterior plane affected the postural control of the lower limbs. In another study by Gribble and Hertel (2004b), which examined the effects of hip and ankle muscle fatigue on postural control during single-leg stance in 4 men and 9 women, they discovered that hip muscle fatigue affected postural control in the anteroposterior and mediolateral planes but ankle muscle fatigue did not produce any significant effect. Although most investigations concluded that ankle muscle fatigue negatively affects postural control; however, some studies reported no changes in postural control after fatigue of the ankle muscles, which may be due to a differences of methods and protocols (Adlerton \& Moritz, 1996; Gribble \& Hertel, 2004b). In addition, some studies have been conducted on the effects of peripheral localized muscle fatigue on postural control using an isokinetic dynamometer; however, studies focusing on the differences in the types of exercise-induced fatigue, such as a comparison of the effects of heel raise and the Harvard step test on functional movements, rarely have been performed. In addition, the effect of post-fatigue recovery on postural control has been poorly documented.

Therefore, the purpose of this study was to investigate the effects of localized muscle fatigue and whole body fatigue on postural control during single-leg stance after impairment induced by heel raise and the Harvard step test in healthy university students.

\section{Methods}

\section{Participants}

Thirty-eight university students (19 men: age, $20.1 \pm 0.2$ yrs; height, $175.0 \pm 5.23 \mathrm{~cm}$; weight, $66.8 \pm 5.6 \mathrm{~kg}$; body mass index, $21.8 \pm 1.7 \mathrm{~kg} / \mathrm{m}^{2}, 19$ women: age, $20.6 \pm 1.1 \mathrm{yrs}$; height, $163.6 \pm 6.7 \mathrm{~cm}$; weight, $58.8 \pm 4.6 \mathrm{~kg}$; body mass index, $22.0 \pm 2.2 \mathrm{~kg} / \mathrm{m}^{2}$ ) in sports-related departments participated in this study. None of the subjects presented with any history of injury of the lower limb that could affect their ability to perform the experiment. Informed consent was obtained from each participant after the purpose of the study and its procedures was explained.

\section{Experimental procedures}

Subjects participated in 2 experimental sessions, separated by approximately 1 week, and were asked to refrain from performing physical exercise and to maintain their regular daily activity level during the study period. During each session, subjects performed a series of single-leg postural tasks prior to, following, and 24 hours after completing 1 of 2 randomly ordered conditions: heel raise or the Harvard step test.

All single-leg postural trials lasted above 70 seconds immediately following each condition, with the patients' eyes open on the platform (Figure 1). During the stance, subjects were instructed to stand barefoot with their preferred leg, with arms crossed at their chest and the knee of the opposite leg in a flexed position, and to visually fixate on the target in front of them. Subjects were instructed to sway as little as possible while single-leg standing.

\section{Fatigue protocol}

The fatigue protocol involved a heel raise and the Harvard step test (Figure 2). The heel raises were performed on a board with an approximately $10^{\circ}$ posterior slope in order to increase the subjects' range of motion. Subjects were 


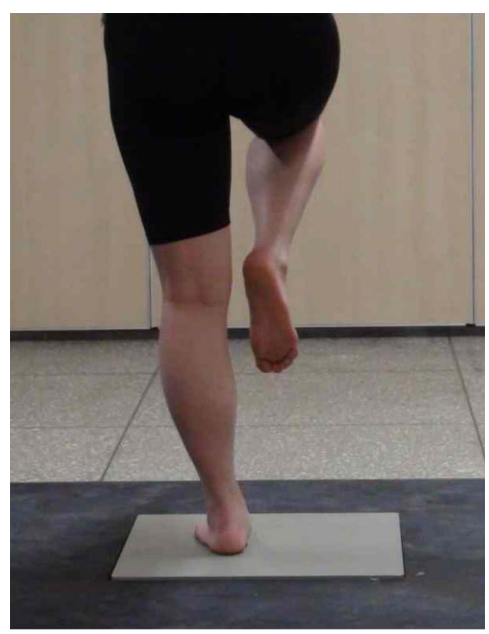

Figure 1. Single-leg stance with eyes open

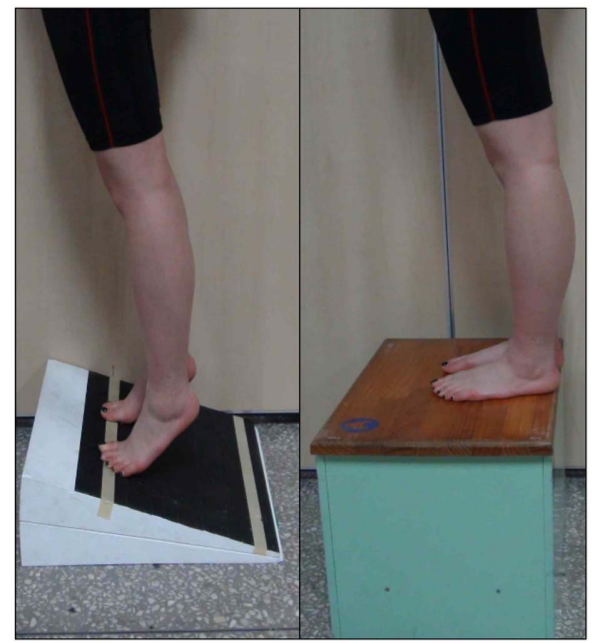

Figure 2. Fatigue protocols (heel raise \& harvard step)

instructed to perform the heel raises at a metronome-paced 40 repetitions $\cdot \mathrm{min}^{-1}$ and the Harvard step test (height, $45 \mathrm{~cm}$ ) at a metronome-paced 30 repetitions $\cdot \min ^{-1}$. A single investigator monitored the performance of the heel raises and the Harvard step test to ensure subjects maintained control during all phases.

Termination of the exercise was determined as the time when the subject was unable to perform throughout the full range of motion, to maintain the established cadence, or when the subject ceased exercise volitionally(American College of Sports Medicine [ACSM], 2006; Springer \& Pincivero, 2009; Vuillerme, Burdet, Isableu, \& Demetz, 2006). Verbal encouragement was provided during all tests that provoked muscle fatigue. To investigate the exercise intensity of each subject, their heart rate was measured with a Polar heart rate monitor (Polar Electro OY, Finland).

\section{Data analysis}

A force platform (AMTI, USA) was used to measure the displacements of the COP during the single-leg stance trials. The COP data were sampled at $100 \mathrm{~Hz}$ and filtered using a fourth-order Butterworth, zero-phase, low-pass filter, with a $10-\mathrm{Hz}$ cut-off frequency. To remove any initial sway that could have occurred under the experimental conditions, the data from 10 seconds to 70 seconds after the start sign were analyzed (Dolye et al., 2007; Raymakers, Samson, \& Verhaar, 2005). The validity and reliability of force platform measurements for stance stability have been reported previously (Hageman, Leibowitz, \& Blanke, 1995). Intraclass correlation coefficients revealed high test-retest reliability for measurements of sway (ICCs $>0.90$; Hageman et al., 1995).

COP data were used to calculate 5 postural variables: the area of the $95 \%$ confidence ellipse $(1)\left(\right.$ in $\left.\mathrm{cm}^{2}\right)$, the mean velocity in the AP and ML planes(2, 3)(in $\mathrm{cm} / \mathrm{s})$, and the mean distance of the root mean square (rms) in the AP and ML planes(4, 5)(in cm). These variables have been assessed in postural control studies and have demonstrated good validity and reliability(Ruhe, Fejer, \& Walker, 2010).

$$
\begin{aligned}
& 95 \% \text { confidence ellipse area }=\pi a b \\
& a=\left[3\left(S D_{A P}^{2}+S D_{M L}^{2}+D\right)\right]^{\frac{1}{2}} \\
& b=\left[3\left(S D_{A P}^{2}+S D_{M L}^{2}-D\right)\right]^{\frac{1}{2}} \\
& D=\left[\left(S D_{A P}^{2}+S D_{M L}^{2}\right)-4\left(S D_{A P}^{2} S D_{M L}^{2}-S D_{A P M L}^{2}\right)\right]^{\frac{1}{2}} \\
& S D_{A P M L}=\frac{1}{N}\left(\sum_{\mathrm{n}}^{N} x_{A P(\mathrm{n})} \mathrm{x}_{\mathrm{ML}(\mathrm{n})}\right) \\
& R M S_{A P}=\left[\frac{1}{N} \sum^{N}\left(x_{A P(n)}-\overline{x_{A P}}\right)^{2}\right]^{\frac{1}{2}} \\
& R M S_{M L}=\left[\frac{1}{N} \sum_{n=1}^{N}\left(x_{M L(n)}-\overline{x_{M L}}\right)^{2}\right]^{\frac{1}{2}} \\
& V_{A P}=\frac{1}{T} \sum_{N=1}^{N-1}\left(\left|x_{A P(n+1)}-x_{A P(n)}\right|\right) \\
& V_{M L}=\frac{1}{T} \sum_{N=1}^{N-1}\left(\left|x_{M L(n+1)}-x_{M L(n)}\right|\right) \\
& N=1
\end{aligned}
$$

\section{Statistical analysis}

Two-way analysis of variance for repeated measurements was used to assess the effects of the time factors (prior to, following, and 24 hours after completing heel raise or the 
Harvard step test) and the fatigue protocol factors (heel raise and the Harvard step test) on the postural variables. Oneway analysis of variance for repeated measurements was performed to assess the effects of the time factors on the postural variables. Post-hoc tests were used, when appropriate, using Bonferroni test and paired $t$-test. The level of significance was set at .05 .

\section{Results}

\section{Results of fatigue protocol}

The results of fatigue protocol are provided in Table 1 . The performed heel raise and the Harvard step test demonstrated significant differences for the exercise duration and maximum heart rate. Therefore, this result showed that their's movement characteristics were different.

Table 1. The results of exercise duration, heart rates and repetitions $(\mathrm{n}=38)$

\begin{tabular}{|c|c|c|c|}
\hline & & Heel raise & Harvard step test \\
\hline \multicolumn{2}{|c|}{$\begin{array}{c}\text { Exercise } \\
\text { duration(min) }\end{array}$} & $2.99 \pm 0.89$ & $13.57 \pm 7.94$ \\
\hline \multirow{2}{*}{$\begin{array}{l}\text { Heart rate } \\
\text { (beats } / \mathrm{m} \text { ) }\end{array}$} & Rest & $71.21 \pm 6.41$ & $71.21 \pm 6.41$ \\
\hline & Max & $110.95 \pm 13.89$ & $185.87 \pm 10.14^{*}$ \\
\hline
\end{tabular}

$*$ is results of paired sample $t$-test; $p<.05$

\section{COP $95 \%$ ellipse area}

The results for all the COP measurements are provided in Table 2 . The $95 \%$ ellipse area results revealed a significant main effect of fatigue $\left(F_{1,37}=3.623, p=.032\right)$ and an interaction effect of fatigue and time $\left(F_{1,37}=4.706, p=.012\right.$; Figure 3 ). The $95 \%$ ellipse area 24 hours after completing the fatigue protocol was smaller than that prior to performing the fatigue protocol $\left(F_{2,111}=3.677, p=.028\right)$, according to the results of the post-hoc test of the Harvard step test. The 95\% ellipse area of the Harvard step test was significantly greater than that of the heel raise $\left(t_{37}=2.320, p=.026\right)$, according to the results of the paired $t$-test at the post.

\section{COP rms distance}

The AP rms distance results showed a significant main effect of fatigue $\left(F_{1,37}=4.749, p=.011\right)$ and an interaction effect of fatigue and time $\left(F_{1,37}=4.565, p=.014\right.$; Figure 4$)$. The AP rms distance following the fatigue protocol was smaller than that prior to performing the fatigue protocol $\left(F_{2,111}=3.181, p=.045\right)$, according to the results of the posthoc test of the heel raise. Furthermore, the AP rms distance 24 hours after completing the fatigue protocol was smaller than that prior to performing the fatigue protocol $\left(F_{2,111}=3.779, p=.026\right)$, based on the results of the post-hoc test of the Harvard step test. The AP rms distance of the Harvard step test was significantly greater than that of the heel raise $\left(t_{37}=2.841, p=.007\right)$, according to the results of

Table 2 The results of center of pressure variables $(n=38)$

\begin{tabular}{|c|c|c|c|c|c|c|}
\hline & & Pre & Post & $24 \mathrm{hrs}$ & $F^{a}$ & Post-hoc \\
\hline \multirow{3}{*}{$\begin{array}{l}\text { 95\% ellipse area } \\
\left(\mathrm{cm}^{2}\right)\end{array}$} & HR & $8.33 \pm 4.00$ & $6.14 \pm 3.48$ & $7.75 \pm 5.26$ & $.310(T)$ & \multirow{3}{*}{ Pre $>24 h r s$} \\
\hline & HST & $8.67 \pm 3.78$ & $7.93 \pm 3.59$ & $6.45 \pm 3.56$ & $3.623(F)^{*}$ & \\
\hline & $t$ & 1.198 & $2.320^{*}$ & 1.256 & $4.706(F \times T)^{*}$ & \\
\hline \multirow{3}{*}{$\begin{array}{l}\text { AP rms } \\
(\mathrm{cm})\end{array}$} & HR & $0.88 \pm 0.21$ & $0.77 \pm 0.16$ & $0.80 \pm 0.20$ & $3.131(T)$ & Pre $>$ Post \\
\hline & HST & $0.90 \pm 0.21$ & $0.87 \pm 0.17$ & $0.78 \pm 0.19$ & $4.749(F)^{*}$ & Pre $>24 \mathrm{hrs}$ \\
\hline & $t$ & 1.140 & $2.841^{*}$ & .575 & $4.565(F \times T)^{*}$ & \\
\hline \multirow{3}{*}{$\begin{array}{l}\text { ML rms } \\
(\mathrm{cm})\end{array}$} & HR & $0.58 \pm 0.09$ & $0.52 \pm 0.09$ & $0.60 \pm 0.10$ & $.029(T)$ & Pre, 24hrs $>$ Post \\
\hline & HST & $0.58 \pm 0.08$ & $0.59 \pm 0.08$ & $0.53 \pm 0.08$ & $2.368(F)$ & Pre, Post $>24 \mathrm{hrs}$ \\
\hline & $t$ & .336 & $5.383^{*}$ & $3.385^{*} .002$ & $22.609(F \times T)^{*}$ & \\
\hline \multirow{3}{*}{$\begin{array}{l}\text { AP velocity } \\
(\mathrm{cm} / \mathrm{s})\end{array}$} & HR & $3.30 \pm 0.79$ & $2.75 \pm 0.73$ & $3.26 \pm 0.91$ & $13.390(T)^{*}$ & Pre, 24hrs $>$ Post \\
\hline & HST & $3.43 \pm 0.85$ & $3.45 \pm 0.94$ & $3.06 \pm 0.90$ & $4.574(F)^{*}$ & \\
\hline & $t$ & $3.756^{*} .001$ & $6.133^{*}$ & 1.716 & $24.081(F \times T)^{*}$ & \\
\hline \multirow{3}{*}{$\begin{array}{l}\text { ML velocity } \\
(\mathrm{cm} / \mathrm{s})\end{array}$} & HR & $3.23 \pm 1.09$ & $2.60 \pm 0.72$ & $2.98 \pm 0.92$ & $5.201(T)^{*}$ & Pre $>$ Post \\
\hline & HST & $3.20 \pm 0.88$ & $3.23 \pm 0.93$ & $2.81 \pm 0.95$ & $4.713(F)^{*}$ & \\
\hline & $t$ & .504 & $5.589^{*}$ & 1.359 & $18.846(F \times T)^{*}$ & \\
\hline
\end{tabular}

$* \mathrm{a}$ is results of two-way ANOVA (fatiguextime) for repeated measures, $t$ is results of paired sample $t$-test (fatigue types); $\mathrm{T}$ means time, $\mathrm{F}$ means fatigue types; AP means anterior-posterior, ML means medial-lateral, rms means root mean squared, HR means Heel Raise, HST means Harvard Step Test, $p<.05$ 


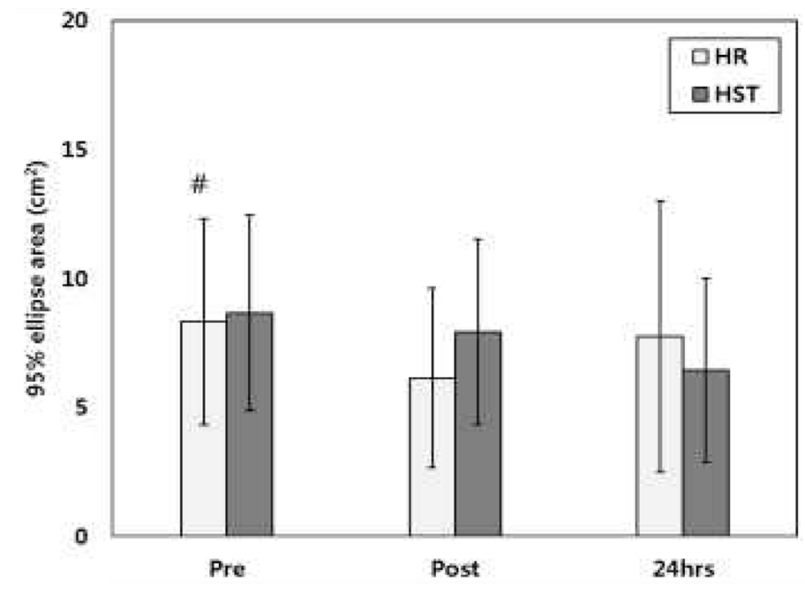

Figure 3. COP $95 \%$ ellipse area. \# is a main effect of fatigue (HR, HST) by two-way repeated measure ANOVA $(p<.05)$

the paired $t$-test at the post.

The ML rms distance results revealed a significant interaction effect of fatigue and time $\left(F_{1,37}=22.609, p=.000\right.$; Figure 5). The ML rms distance following the fatigue protocol was smaller than that prior to and 24 hours after completing the fatigue protocol $\left(F_{2,111}=6.145, p=.003\right)$, according to the results of the post-hoc test of the heel raise. In addition, the ML rms distance 24 hours after completing the fatigue protocol was smaller than that prior to and following the fatigue $\operatorname{protocol}\left(F_{2,111}=6.977, p=.001\right)$, based on the results of the post-hoc test of the Harvard step test. According to the results of the paired $t$-test, the ML rms distance of the Harvard step test was significantly greater than that of the heel raise $\left(t_{37}=5.383, p=.000\right)$ at the Post, while the AP rms distance of the Harvard step test was significantly smaller than that of the heel raise $\left(t_{37}=3.385, p=.002\right)$ at the $24 \mathrm{hrs}$.

\section{COP velocity}

The AP velocity results showed a significant main effect of time $\left(F_{1,37}=13.390, p=.001\right)$, main effect of fatigue $\left(F_{1,37}=4.574, p=.013\right)$, and interaction effect of fatigue and time $\left(F_{1,37}=24.081, p=.000\right.$; Figure 6$)$. The AP velocity following the fatigue protocol was smaller than that prior to and 24 hours after completing the fatigue protocol $\left(F_{2,111}=5.476, p=.005\right)$, based on the results of the post-hoc test of the heel raise. The AP velocity of the Harvard step test was significantly greater than that of the heel raise, according to the results of the paired $t$-test at the Pre $\left(t_{37}=3.756, p=.001\right)$ and the Post $\left(t_{37}=6.133, p=.000\right)$.

The ML velocity results revealed a significant main effect of time $\left(F_{1,37}=5.201, p=.028\right)$, main effect of fatigue $\left(F_{1,37}=4.713, p=.012\right)$, and interaction effect of fatigue and time $\left(F_{1,37}=18.846, p=.000\right.$; Figure 7$)$. The ML velocity

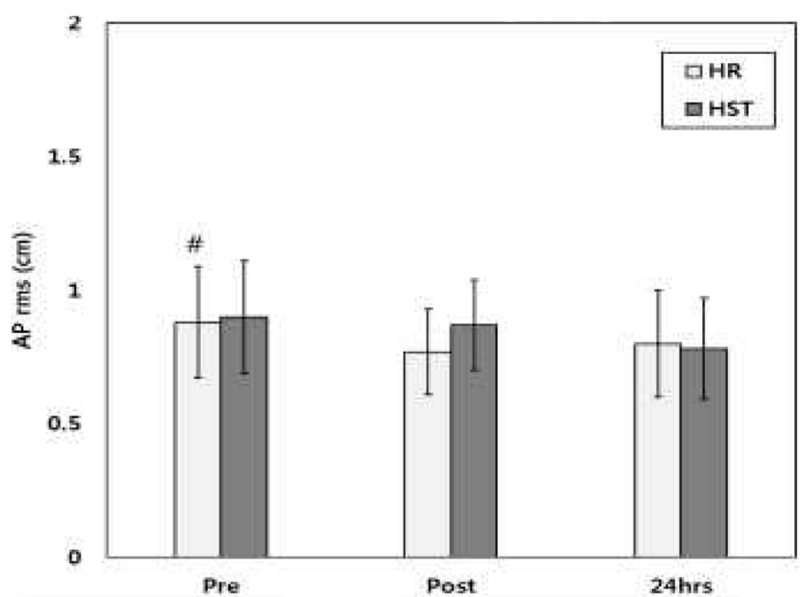

Figure 4. COP rms distance in the AP. \# is a main effect of fatigue (HR, HST) by two-way repeated measure ANOVA $(p<.05)$

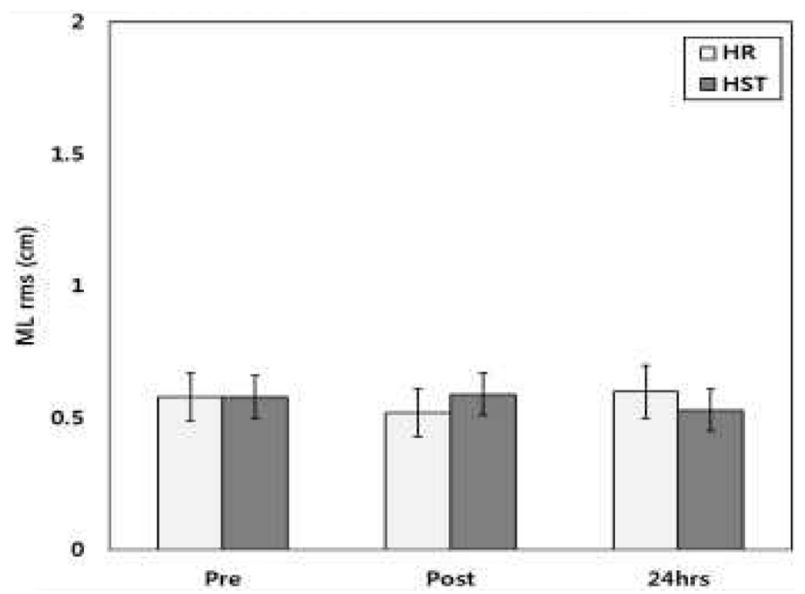

Figure 5. COP rms distance in the ML. \# is a main effect of fatigue (HR, HST) by two-way repeated measure ANOVA $(p<.05)$

following the fatigue protocol was smaller than that prior to performing the fatigue protocol $\left(F_{2,111}=4.403, p=.014\right)$, according to the results of the post-hoc test of the heel raise. The ML velocity of the Harvard step test was significantly greater than that of the heel raise $\left(t_{37}=5.589, p=.000\right)$, according to the results of the paired $t$-test at the post.

\section{Discussion}

The purpose of this study was to investigate the effects of localized muscle fatigue and whole body fatigue on postural control during single-leg stance after impairment induced by heel raise and the Harvard step test in healthy university students.

The International Society for Posture and Gait Research (ISPGR) suggests using the rms distance and velocity in the AP and ML planes of the COP to evaluate postural control 


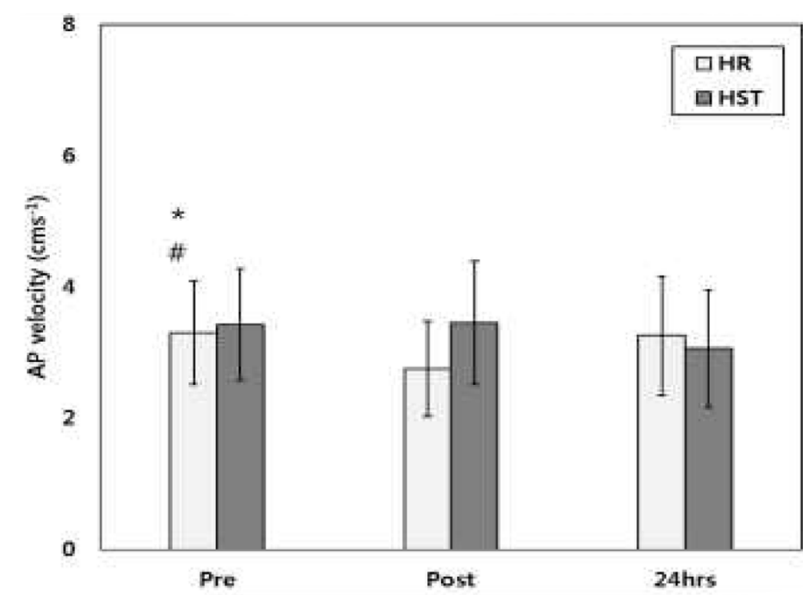

Figure 6. COP velocity in the AP. * is a main effect of time (Pre, Post, 24hrs) by two-way repeated measure ANOVA $(p<.05)$; \# is a main effect of fatigue (HR, HST) by two-way repeated measure ANOVA $(p<.05)$

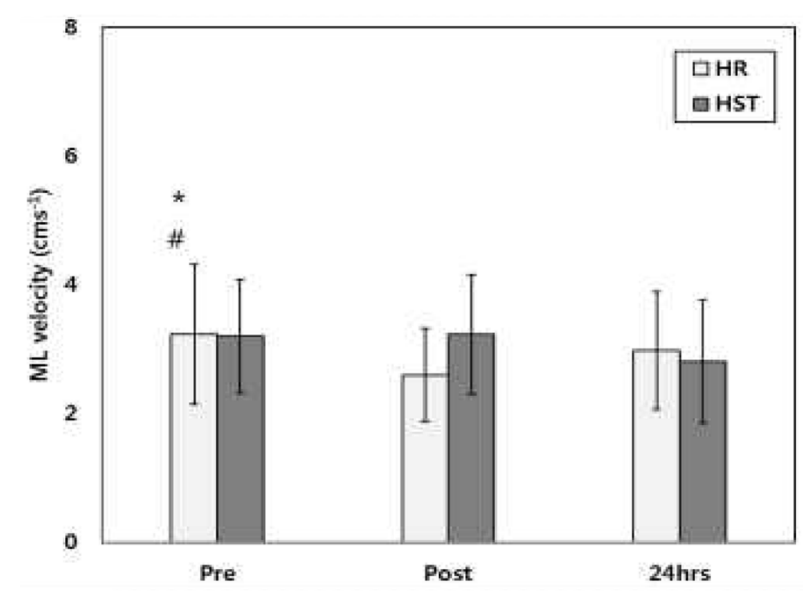

Figure 7. COP velocity in the AP. * is a main effect of time(Pre, Post, 24hrs) by two-way repeated measure ANOVA $(p<.05)$; \# is a main effect of fatigue (HR, HST) by two-way repeated measure ANOVA $(p<.05)$

by using the COP trajectory of the ground reaction force system (Kapteyn et al., 1983), whereas current studies investigating postural control with COP used the $95 \%$ confidence ellipse area more frequently than other time domain variables (Dolye et al., 2007; Prieto, Myklebust, J. B, Hoffmann, Lovett, \& Myklebust, B. N., 1996; Youm \& Kim, 2012; Rocchi, Chiari, \& Cappello, 2004). Therefore, the COP variables in this study were limited to AP and ML rms distance, AP and ML velocity, and 95\% confidence ellipse area.

The major findings of this study were that heel raise exercise-induced fatigue affected the rms distance and velocity in the AP and ML planes of the COP, and that these variables decreased significantly following the fatigue protocol
(Youm \& Kim, 2012). Furthermore, we observed that Harvard step exercise-induced fatigue affected the rms distances in the AP and ML planes and the 95\% confidence ellipse area of the COP, and that these variables decreased significantly 24 hours after completing the fatigue protocol.

Yaggie and McGregor (2002) measured postural control during single-leg stance after plantar flexor and dorsiflexor fatigue in 24 healthy men, and reported that isokinetic fatigue significantly increased the sway parameters. Gribble and Hertel (2004a) examined the effects of ankle, knee, and hip joint fatigue on postural control during single-leg stance in 4 men and 10 women, and showed that fatigue of the knee and hip muscles led to significantly greater velocity of the COP in the ML plane, whereas fatigue of the ankle muscle did not. They also reported that the AP factors were affected by fatigue of the ankle, knee, and hip muscles. Gimmon et al. (2011) measured postural control during upright stance after the ankle plantar flexor contractions as possible until exhaustion, in 3 men and 7 women, reported that localized plantar flexor fatigue caused impairment to postural control in the sagittal plane. As such, a decrease in performance could be related to fatigue affecting the proprioceptive feedback from afferent pathways (Gimmon et al., 2011).

In addition, Gribble and Hertel (2004b) investigated the effects of hip and ankle muscle fatigue on postural control during single-leg stance in 4 men and 9 women, and found that fatigue of the hip muscle led to significantly greater velocity of the COP in the AP and ML planes, whereas fatigue of the ankle muscle did not significantly impair postural control in either plane. According to Springer and Pincivero (2009), who studied the effects of localized muscle and whole-body fatigue on balance during single-leg stance in 10 men and 10 women, both localized muscle fatigue and whole-body fatigue produced significant increases in $\mathrm{ML}$ and total sway. Total sway increased more significantly following the localized muscle fatigue protocol than after the whole-body fatigue protocol. Their results were somewhat different than the results of this study.

The increased COP variables generally were interpreted as decreased stability or poor postural control (Adlerton, Moritz, \& Moe-Nilssen, 2003; Cobeil et al., 2003; Raymakers et al., 2005; Prieto et al., 1996). However, the variables that were reduced significantly after heel raise (following the fatigue protocol) and the Harvard step test (24 hours after completing the fatigue protocol), unlike the previous results (Gribble \& Hertel, 2004a; Gribble \& Hertel, 2004b, Shin \& Youm, 2011; Springer \& Pincivero, 2009; Yaggie \& McGregor, 2002; Youm \& Kim, 2012).

Adlerton et al. (2003) examined the effects of heel raise fatigue on postural control during single-leg stance in 23 healthy women, and found that the COP velocity and frequency decreased significantly. According to the results of 
this study, the reduction in most COP variables after the fatigue protocols can be interpreted as a change in the postural control strategy from ankle to hip, which is similar to the conclusions determined by Adlerton et al. (2003), and Panzer, Bandinelli, and Hallett (1995). Generally, there are 2 common strategies for control of body sway during standing: the ankle strategy and the hip strategy (Winter, 1995). Under normal circumstances, the ankle strategy is effective in maintaining static standing and responding to a small sway, but, when the muscle around the ankle experiences fatigue, posture is regulated by relying on the muscle around the hip joint; this is called the hip strategy. On the other hand, if the muscle around the hip joint experiences fatigue, the opposite situation occurs, which is called the ankle strategy (Bizid et al., 2009; Gribble \& Hertel, 2004a). Therefore, single-leg stance is controlled by a combination of the ankle and hip strategies (Bisson, McEwen, Lajoie, \& Bilodeau, 2011; Riemann, Myers, \& Lephart, 2003). Some have questioned whether $\mathrm{COP}$ measurements are valid indicators of postural control (Adlerton et al., 2003; Winter, 1995; Moe-Nilssen, 1998) because changes in the COP trajectory are not always accompanied by corresponding changes in the position of the COM. Thus, this study suggests that changes in the postural control strategy may have occurred after the fatigue protocols during single-leg stance.

Corrective contractions in response to small sways are occurring constantly. However, fatigue slows neural transmission due to the reduced ability to efficiently create compensatory contractions about a joint. A larger variability in joint motion due to the lack of corrective muscle actions can result in diminished postural control ability (Gribble \& Hertel, 2004a). In addition, when postural sway occurs at each joint, fatigue possibly can inhibit the ability of the surrounding muscles to react to small sways (Gribble \& Hertel, 2004a). For example, when the ankle muscle becomes fatigued, it is possible that afferent conduction velocities still remain constant in response to perturbation. Although efferently signaled compensatory contractions are reduced due to the induced fatigue, the afferent conduction rate is not reduced by responding to the sway. A lack of compensatory contraction at the ankle tends to reduce the rms and velocity of the COP(Gribble \& Hertel, 2004a). Therefore, ankle muscle fatigue reduces COP rms and velocity, similar to the results seen in this study after completing the fatigue protocols(Youm \& Kim, 2012).

Vuillerme, Burdet, Isableu, \& Demetz (2001) showed that, to some extent, vision can compensate for muscle fatigue of the calf muscles. Therefore, the availability of vision allowed the subjects in this study to immediately cope with the destabilizing effect induced by muscle fatigue. Moreover, Bission et al. (2011) reported that a decrease in ankle proprioception may not be sufficient to affect sway velocity when vision is available. Consequently, vision can attenuate the postural deficits associated with fatigue of the ankle plantar flexors. In this study, the reliance on vision was possible because subjects were tested with their eyes open.

Interestingly, in this study, heel raise exercise-induced fatigue reduced the COP variables; however, there was no significant increase in the variables due to Harvard step exercise-induced fatigue. In addition, Harvard step exercise-induced fatigue decreased 24 hours after completing the fatigue protocol. As in previous studies, the effects of heel raise exercise-induced fatigue in this study were shortlived (Adlerton et al., 2003; Bisson et al., 2011; Yaggie \& McGregor, 2002). However, Harvard step exercise-induced fatigue in this study was different; the effects not only were observed immediately following the protocol, but also were seen 24 hours later. Therefore, it is believed that there is specificity in the process of treating exercise-induced fatigue (Cairns, Knicker, Thompson, \& Sjøgaard, 2005; Enoka \& Stuart, 1992; Bigland-Ritchie, Rice, Garland, \& Walsh, 1995).

\section{Conclusion}

The purpose of this study was to investigate the effects of fatigue and recovery on postural control during single-leg stance after impairment induced by heel raise and the Harvard step test in healthy university students. The findings of this study indicated that heel raise exercise-induced fatigue affected the rms distance and velocity in the AP and ML planes of the COP, and that these variables decreased significantly following the fatigue protocol. We also observed that Harvard step exercise-induced fatigue affected the rms distance in the AP and ML planes, and the $95 \%$ confidence ellipse area of the COP, and that these variables decreased significantly 24 hours after completing the fatigue protocol. In conclusion, this study showed that both heel raise exercise- and Harvard step exercise-induced fatigue affects postural control during single-leg stance in AP and ML planes. Furthermore, this study suggests that changes in the postural control strategy may have occurred after the fatigue protocols during single-leg stance. Also vision can attenuate the postural deficits associated with the fatigues. In order to clarify these results, further studies using other equipment and variables are necessary.

\section{References}

American College of Sports Medicine (2006). Guidelines for exercise testing and prescription. Baltimore: Lippincott Williams \& Wilkins.

Adlerton, A. K., \& Moritz, U. (1996). Does calf-muscle fatigue affect standing balance?. Scandinavian Journal of Science in Sports, 6, 211-215. 
Adlerton, A. K., Moritz, U., \& Moe-Nilssen, R. (2003). Force plate and accelerometer measures for evaluating the effect of muscle fatigue on postural control during one-legged stance. Physiotherapy Research International, 8, 187-199.

Allen, D. G., \& Westerblad, H. (2001). Role of phosphate and calcium stores in muscle fatigue. The Journal of Physiology, 536, 657-665.

Bigland-Ritchie, B., Rice, C. L., Garland, S. J., \& Walsh, M. L. (1995). Task-dependent factors in fatigue of human voluntary contractions. Advances in Experimental Medicine And Biology, 384, 361-380.

Bisson, E. J., McEwen, D., Lajoie, Y., \& Bilodeau, M. (2011). Effects of ankle and hip muscle fatigue on postural sway and attentional demands during unipedal stance. Gait and Posture, 33(1), 83-87.

Bizid, R., Margnes, E., Francois, Y., Jully, J. L., Gonzalez, G., \& Dupui, P. (2009). Effects of knee and ankle muscle fatigue on postural control in the unipedal stance. European Journal of Applied Physiology, 106, 375-380.

Bohannon, R. W., \& Leary, K. M. (1995). Standing balance and function over the course of acute rehabilitation. Archives of Physical Medicine and Rehabilitation, 76, 994-996.

Cairns, S. P., Knicker, A. J., Thompson, M. W., \& Sjøgaard, G. (2005). Evaluation of models used to study neuromuscular fatigue. Exercise and Sport Science Reviews, 33(1), 9-16.

Christina, K. A., White, S. C., \& Gilchrist, L. A. (2001). Effect of localized muscle fatigue on vertical ground reaction forces and ankle joint motion during running. Human Movement Science, 20, 257-276.

Cobeil, P., Blouin, J. S., Bégin, F., Nougier, V., \& Teasdale, N. (2003). Pertubation of the postural control system induced by muscular fatigue. Gait and Posture, 18, 92-100.

Dolye, R. J., Hsiao-Wecksler, E. T., Ragan, B. G., \& Rosengren, K. S. (2007). Generalizability of center of pressure measures of quiet standing. Gait \& Posture, 25, 166-171.

Enoka, R. M., \& Stuart, D. G. (1992). Neurobiology of muscle fatigue. Journal of Applied Physiology, 72(5), 1631-1648.

Frzovic, D., Morris, M. E., \& Vowels, L. (2000). Clinical tests of standing balance: performance of persons with multiple sclerosis. Archives of Physical Medicine and Rehabilitation, 81, 215-221.

Gimmon, Y., Riemer, R., Oddsson, L., \& Melzer, I. (2011). The effect of plantar flexor muscle fatigue on postural control. Journal of Electromyography and Kinesiology, 21, 922-928.

Giannesini, B., Cozzone, P. J., \& Bendahan, D. (2003). Non-invasive investigations of muscular fatigue: metabolic and electromyographic components. Biochimie, 85, 873-883.

Gribble, P. A., \& Hertel, J. (2004a). Effect of lower-extremity muscle fatigue on postural control. Archives of Physical Medicine And Rehabilitation, 85(4), 589-592.

Gribble, P. A., \& Hertel, J. (2004b). Effect of hip and ankle muscle fatigue on unipedal postural control. Journal of Electromyography and Kinesiology, 14, 641-646.

Hageman, P. A., Leibowitz, J. M., \& Blanke, D. (1995). Age and gender effects on postural control measures. Archives of Physical Medicine and Rehabilitation, 76(10), 961-965.

Hall, C. M., \& Brody, L. T. (1999). Therapeutic Exercise: Moving Toward Function. Baltimore: Lippincott Williams \&
Wilkins.

Jonsson, E., Seiger, A., \& Hirschfeld, H. (2004). One-leg stance in healthy young and elderly adults: a measure of postural steadiness?. Clinical Biomechanics, 19, 688-694.

Kapteyn, T. S., Bles, W., Njiokiktjien, C. J., Kodde, L., Massen, C. H., \& Mol, J. M. F. (1983). Standardization in platform stabilometry being part of posturography. Agressologie, 24, 321-326.

Letafatkar, K., Alizadeh, M. H., \& Kordi, M. R. (2009). The effect of exhausting exercise induced fatigue on the double-leg balance of elite male athletes. Journal of Social Sciences, 5(4), 445-451.

Lin, D., Nussbaum, M. A., Seol, H., Singh, N. B., Madigan, M. L., \& Wojcik, L. A. (2009). Acute effects of localized muscle fatigue on postural control and patterns of recovery during upright stance: influence of fatigue location and age. European Journal of Applied Physiology, 106(3), 425-434.

Lundin, T. M., Feuerbach, J. W., \& Grabiner, M. D. (1993). Effect of plantar flexor and dorsiflexor fatigue on unilateral postural control. Journal of Applied Biomechanics, 9(2), 191-201.

Massion, J. (1994). Postural control system. Current Opinion in Neurobiology, 4(6), 877-887.

Moe-Nilssen, R. (1998). Test-retest reliability of trunk accelerometry during standing and walking. Archives of physical medicine and rehabilitation, 79(11), 1377-1385.

Panzer, V. P., Bandinelli, S., \& Hallett, M. (1995). Biomechanical assessment of quiet standing and changes associated with aging. Archives of Physical Medicine and Rehabilitation, 76(2), 151-157.

Prieto, T. E., Myklebust, J. B., Hoffmann, R. G., Lovett, E. G., \& Myklebust, B. M. (1996). Measures of postural steadiness: differences between healthy young and elderly adults. IEEE Transactions on Biomedical Engineering, 43(9), 956-966.

Raymakers, J. A., Samson, M. M., \& Verhaar, H. J. J. (2005). The assessment of body sway and the choice of the stability parameter(s). Gait and Posture, 21, 48-58.

Riemann, B. L., Myers, J. B., \& Lephart, S. M. (2003). Comparison of the ankle, knee, hip, and trunk corrective action shown during single-leg stance on firm, foam, and multiaxial surfaces. Archives of Physical Medicine and Rehabilitation, 84, 90-95.

Rocchi, L., Chiari, L., \& Cappello, A. (2004). Feature selection of stabilometric parameters based on principal component analysis. Medical \& Biological Engineering \& Computing, 42, 71-79.

Rozzi, S. L., Lephart, S. M., Gear, W. S., \& Fu, F. H.(1999). Knee joint laxity and neuromuscular characteristics of male and female soccer and basketball players. The American Journal of Sports Medicine, 27(3), 312-319.

Ruhe, A., Fejer, R. \& Walker, B. (2010). The test-retest reliability of centre of pressure measures in bipedal static task conditionsa systematic review of the literature. Gait and Posture, 32, 436-445.

Shin, J. D. \& Youm, C. H. (2011). Effects of heel raise and harvard step exercise-induced fatigue on single-leg stance postural control. The Korean Journal of physical Education, 50(3), 581-591.

Shumway-Cook, A. \& Woollacott, M. H. (2000). Motor control: 
Theory and practical applications. 2nd edition. Baltimore: Lippincott Williams \& Wilkins.

Springer, B. K. \& Pincivero, D. M. (2009). The effects of localized muscle and whole-body fatigue on single-leg balance between healthy men and women. Gait and Posture, 30, 50-54.

Urabe, Y., Kobayashi, R., Sumida, S., Tanaka, K., Yosida, N., Nishiwaki, G. A., Tsutsumi, E., \& Ochi, N. (2005). Eletromyographic analysis of the knee during jump landing in male and female athletes. Knee, 12, 129-134.

Vuillerme, N, Nougier, V., \& Prieur, J. (2001). Can vision compensate for a lower limbs muscular fatigue for controlling posture in humans?. Neuroscience Letters, 308, 103-106.

Vuillerme, N, Burdet, C., Isableu, B., \& Demetz, S. (2006). The magnitude of the effect of calf muscles fatigue on postural control during bipedal quiet standing with vision depends on the eye-visual target distance. Gait and Posture, 24, 169172 .

Winter, D. A. (1995). Human balance and posture control during standing and walking. Gait and Posture, 3, 193-214.

Yaggie, J. A. \& McGregor, S. J. (2002). Effects of isokinetic ankle fatigue on the maintenance of balance and postural limits. Archives of Physical Medicine and Rehabilitation, 83(2), 224-228.

Youm, C. H. \& Kim, T. H. (2012). Effects of induced fatigue of ankle joint muscle on the capability and recovery of postural control during single-leg stance. Korean Journal of Sport Biomechanics, 22(2), 219-228. 